\title{
Protein modeling to assess the pathogenicity of rare variants of SERPINA1 in patients suspected of having Alpha 1 Antitrypsin Deficiency
}

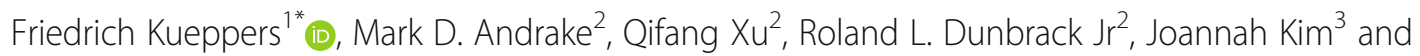
Christopher L. Sanders ${ }^{3}$

\begin{abstract}
Background: Alpha 1 Antitrypsin (AAT) is a key serum proteinase inhibitor encoded by SERPINA1. Sequence variants of the gene can cause Alpha 1 Antitrypsin Deficiency (AATD), a condition associated with lung and liver disease. The majority of AATD cases are caused by the ' $Z$ ' and ' $S$ ' variants - single-nucleotide variations (SNVs) that result in amino acid substitutions of E342K and E264V. However, SERPINA1 is highly polymorphic, with numerous potentially clinically relevant variants reported. Novel variants continue to be discovered, and without reports of pathogenicity, it can be difficult for clinicians to determine the best course of treatment.
\end{abstract}

Methods: We assessed the utility of next-generation sequencing (NGS) and predictive computational analysis to guide the diagnosis of patients suspected of having AATD. Blood samples on serum separator cards were submitted to the DNA 1 Advanced Screening Program (Biocerna LLC, Fulton, Maryland, USA) by physicians whose patients were suspected of having AATD. Laboratory analyses included quantification of serum AAT levels, qualitative analysis by isoelectric focusing, and targeted genotyping and NGS of the SERPINA1 gene. Molecular modeling software UCSF Chimera (University College of San Francisco, CA) was used to visualize the positions of amino acid changes as a result of rare/novel SNVs. Predictive software was used to assess the potential pathogenicity of these variants; methods included a support vector machine (SVM) program, PolyPhen-2 (Harvard University, Cambridge, MA), and FoldX (Centre for Genomic Regulation, Barcelona, Spain).

Results: Samples from 23 patients were analyzed; 21 rare/novel sequence variants were identified by NGS, including splice variants $(n=2)$, base pair deletions $(n=1)$, stop codon insertions $(n=2)$, and SNVs $(n=16)$. Computational modeling of protein structures caused by the novel SNVs showed that 8 were probably deleterious, and two were possibly deleterious. For the majority of probably/possibly deleterious SNVs (I50N, P289S, M385T, M221T, D341V, V210E, P369H, V333M and A142D), the mechanism is probably via disruption of the packed hydrophobic core of AAT. Several deleterious variants occurred in combination with more common deficiency alleles, resulting in very low AAT levels.

Conclusions: NGS and computational modeling are useful tools that can facilitate earlier, more precise diagnosis, and consideration for AAT therapy in AATD.

Keywords: Alpha 1 Antitrypsin Deficiency, SERPINA1, Rare variants, Protein modeling

\footnotetext{
* Correspondence: Friedrich.Kueppers@tuhs.temple.edu

'Department of Thoracic Medicine and Surgery, Lewis Katz School of

Medicine, Temple University, Philadelphia, PA, USA

Full list of author information is available at the end of the article
}

(c) The Author(s). 2019 Open Access This article is distributed under the terms of the Creative Commons Attribution 4.0 International License (http://creativecommons.org/licenses/by/4.0/), which permits unrestricted use, distribution, and reproduction in any medium, provided you give appropriate credit to the original author(s) and the source, provide a link to the Creative Commons license, and indicate if changes were made. The Creative Commons Public Domain Dedication waiver (http://creativecommons.org/publicdomain/zero/1.0/) applies to the data made available in this article, unless otherwise stated. 


\section{Background}

Alpha 1 Antitrypsin (AAT) is a glycoprotein normally present in human blood at a concentration between 90 and $180 \mathrm{mg} / \mathrm{dL}$ [1]. It is encoded by the SERPINA1 gene that is located on the long arm of chromosome 14 (cytogenetic location: 14q32.13); the gene encompasses 12.2 $\mathrm{kb}$, containing 4 exons and 3 introns [2]. AAT is an effective inhibitor of serine proteinases, in particular leukocytic elastase; in this capacity it exerts a protective function on various tissues, especially the lungs, against proteolytic/elastolytic damage [3].

AAT is a highly polymorphic protein; over 70 sequence variants have been reported to be clinically significant and over 500 single-nucleotide variations (SNVs) identified in mutation databases. Some variants are common in certain populations such that their frequency may be maintained by a heterozygous selective advantage [4]. Common alleles that fit this definition, including $\mathrm{PI} * \mathrm{Z}$ and $\mathrm{PI}{ }^{*} \mathrm{~S}$, are frequent in Northern Europe and Spain/Portugal respectively [5].

The nomenclature ( $\mathrm{Z}, \mathrm{S}, \mathrm{M}$ etc.) refers to a lettering system in which the normal common allele is designated $\mathrm{PI}^{*} \mathrm{M}$, and other letters refer to the isoelectric point of the protein in a $\mathrm{pH}$ gradient established by isoelectric focusing (IEF) - a common method used to identify AAT variants [1]. Certain relatively common variants, in particular PI*Z and S, are associated with low levels of AAT in the circulation [1]. The $\mathrm{Z}$ and $\mathrm{S}$ alleles are caused by E342K and E264V substitutions, respectively; both cause misfolding and polymerization (to a lesser extent with the $\mathrm{S}$ allele) of AAT [2]. The $\mathrm{Z}$ mutation also results in retention of polymerized AAT in hepatocytes, leading to severe deficiency and liver disease, and is of special clinical interest.

Among patients of European ancestry with chronic obstructive pulmonary disease (COPD; including emphysema), 1-3\% have been found to have Alpha 1 Antitrypsin Deficiency (AATD), usually due to homozygosity for PI*Z [6]. There are, however, less common deficiency alleles that can also be associated with reduced AAT levels and lung disease [7-9]. As testing and screening become more widely used, more variants associated with low AAT levels continue to be uncovered [10]. Primarily, the improved identification of rare/novel variants is due to the increased use of DNA sequencing. In particular, next-generation sequencing (NGS), a far higher-throughput technology than Sanger sequencing [11], has the potential to improve the diagnosis of AATD through the enhanced detection of rare/novel variants [12].

We report a number of rare/novel SERPINA1 sequence variants detected with the use of NGS in a USwide AATD targeted detection program. To characterize the potential deleterious effects of these variants, we utilized a number of molecular modeling analyses. Our aim was to cover the whole spectrum from the nucleotide base change to the altered protein structure, and predict the clinical consequences to the patient.

\section{Methods \\ Subjects}

Patients were recruited from the Lewis Katz School of Medicine, Temple University, Philadelphia, Pennsylvania $(n=4)$ or through nationwide physician referral to the $\mathrm{DNA}_{1}$ Advanced Alpha-1 Screening ${ }^{\mathrm{Tm}}$ program (developed and performed by Biocerna LLC, Fulton, Maryland, USA, on behalf of CSL Behring, King of Prussia, Pennsylvania, USA; $n=19$ ). Blood samples on serum separator cards were collected through routine clinical testing by the treating physician and sent to Biocerna for AATD screening.

Patients were included in this study if discordance existed between the patient's AAT level and the targeted genotyping results. For these patients, NGS was used to identify rare or potentially novel genetic variants. Consent for use of laboratory data for research purposes was provided by all patients included in this study. The study was approved by the Institutional Review Board of Temple University, Philadelphia, PA.

\section{Laboratory analyses}

Data on antigenic serum AAT and c-reactive protein levels, AAT phenotype by IEF, and genetic analyses by targeted real-time polymerase chain reaction and NGS were collected for patients included in this study.

Serum AAT levels were assessed in all patients. For the four patients referred from Lewis Katz School of Medicine, quantitative analysis of antigenic serum AAT was performed by radial immunodiffusion (normal range: $150-400 \mathrm{mg} / \mathrm{dL}$ ) at Temple University. For the remaining patients, antigenic AAT and CRP levels were assessed using immunoturbidimetry (normal range: 90 $200 \mathrm{mg} / \mathrm{dL}$ and $<5 \mathrm{mg} / \mathrm{L}$, respectively) [Roche ${ }^{\mathrm{Tw}}$ AAT2 and C-Reactive Protein gen 3 immunoassay; Basel, Switzerland] at Biocerna.

All genetic and IEF analyses were performed centrally at Biocerna. Initial qualitative assessment of AATD genotype was by real-time polymerase chain reaction targeted genotyping (TaqMan: Thermo Fisher Scientific, Waltham, MA). Phenotype was investigated using IEF (Hydragel 18 A1AT IEF isofocusing kit, Sebia USA, Norcross, GA).

NGS methodology includes sequencing of SERPINA1 $5^{\prime}$ and $3^{\prime}$ untranslated regions (UTRs), the promotor region, coding exons, introns, and splice sites. Specific target regions of the SERPINA1 gene were amplified using the Ion AmpliSeq ${ }^{\mathrm{Tm}}$ Custom Primer Pools (Thermo Fisher Scientific). The primer pools included a total of 52 amplicons containing unique PCR primers to amplify specific target sequences in each template DNA. Library preparation was performed using Ion AmpliSeq ${ }^{\text {Tix }}$ Library Kit 2.0-96 LV (Thermo Fisher Scientific). Sample 
identity was maintained using unique Ion Xpress ${ }^{\mathrm{Tw}}$ Barcode Adapters (Thermo Fisher Scientific), which allowed for multiplexed sequencing analysis. Emulsion PCR was used to clonally amplify the library DNA onto the Ion Sphere $^{\text {Tix }}$ Particles (ISP). Following ISP template amplification, the ISP-enriched template-positive library was loaded onto an Ion $314^{\text {Tu }}$ Chip Kit v2 (Thermo Fisher Scientific). The Ion PGM instrument was used to sequence the combined library.

\section{Computational modeling and variant predictions}

To visualize and map the locations of sequence variants in the AAT proteins, molecular modeling software UCSF Chimera (University College of San Francisco, CA) was used (Fig. 1). A linear diagram of the AAT amino acid sequence with mutation locations was also prepared (Fig. 2) using the software package ESPript [13].

\section{Predicting pathogenicity}

For all missense SNVs, NGS sequencing information was inputted into a support vector machine (SVM) model. This model combines multiple features, including both sequence- and structure-based information, to calculate the probability that a given missense change is pathogenic [14]. The SVM score is in the range of zero to 1.0, with a threshold for a deleterious change set at a value of 0.5 and above. Further details concerning the SVM model utilized are outlined in the Additional file 1.

In addition to the SVM predictions, two other computational predictors of pathogenicity were utilized. First, Gibbs free energy changes $(\Delta \Delta G)$ associated with amino acid substitutions were calculated using the PositionScan function of the FoldX suite [Centre for Genomic Regulation, Barcelona, Spain] [15]. $\Delta \Delta \mathrm{G}$ is the difference in free energy (in $\mathrm{kcal} / \mathrm{mol}$ ) between a mutant and wildtype protein. A mutation with $\Delta \Delta G>0$ will destabilize the structure, while a mutation with negative $\Delta \Delta \mathrm{G}$ stabilizes the structure. A common threshold used to indicate that a mutation has a significant destabilizing effect is $\Delta \Delta G>$ $1 \mathrm{kcal} / \mathrm{mol}$ [16], and was therefore set as the threshold for pathogenicity in the present report. Second, PolyPhen-2 program (http://genetics.bwh.harvard.edu/ pph2/index.shtml; version 2.2.2, Harvard University, Cambridge, MA) was also used to predict the pathogenicity of all missense SNVs. PolyPhen-2 uses an iterative greedy algorithm, informed by exposure to known damaging and non-damaging SNVs, and calculates the Naïve Bayes posterior probability that a given mutation is damaging [17]. The Polyphen-2 score, also with a range of zero to 1.0 (but often stated as a percentage), has a qualitative ternary classification. Scores of $0.85,0.85-$ 0.15 , and 0.15 are typically coded as "probably damaging", "possibly damaging", and "benign", respectively.
Scores for all three predictive methods (SVM, FoldX, and PolyPhen-2) were grouped into the following classifications: probably deleterious (all three predictions as deleterious), possibly deleterious (two of the three predictions as deleterious), possibly neutral (only one of the three predictions as deleterious), or probably neutral (none of the three predictions as deleterious).

\section{Benchmarking analysis of SVM predictions}

We confirmed the effectiveness of the SVM method by performing benchmarking analysis against two datasets of known human SERPINA1 pathogenic and benign variants sourced from ClinVar [18], and a third dataset composed of primate neutral variants (owing to the low number of benign human variants identified $[N=6]$ ). To build the dataset of primate neutral variants, we ran PSIBLAST with the Alpha 1 Antitrypsin (SERPINA1, A1AT_HUMAN) sequence as a query against a database of primate sequences from Uniprot (http://www.uniprot. org/). For each alignment, we identified all sequence differences between the human and primate sequence and filtered out sequence variants that were not surrounded by 2 conserved residues on either side (human and primate identical) and those adjacent to gaps within 3 residues. For each mutant, we used the search result with the highest sequence identity for that variant. In this manner we chose sequence variants that exist in the closest homologues first. We also checked the contacts of the human residue for each mutation in SERPINA1 structure PDB: $3 \mathrm{NE} 4$ and filtered out sequence variants with one or more different contact residues. A contact is defined as a residue with at least one atomic distance less than $5 \AA$. This resulted in 35 neutral sequence variants garnered from primates with greater than $90 \%$ sequence identity.

\section{Measurements of binary prediction of SERPINA1 variants}

To further compare the accuracy of SVM predictions vs. PolyPhen-2, a number of statistical parameters were calculated. From the benchmarking data, we are able to obtain the number of true positives (TP), false positives (FP), true negatives (TN), and false negatives (FN). From these, we calculated the true positive rate (TPR), true negative rate (TNR), positive predictive value (PPV), and negative predictive value (NPV).

True positive rate (TPR), or sensitivity: The proportion of experimental positives that are correctly predicted.

$$
\mathrm{TPR}=\frac{\mathrm{TP}}{\mathrm{TP}+\mathrm{FN}}
$$

True negative rate (TNR), or specificity: The proportion of experimental negatives that are predicted correctly. 

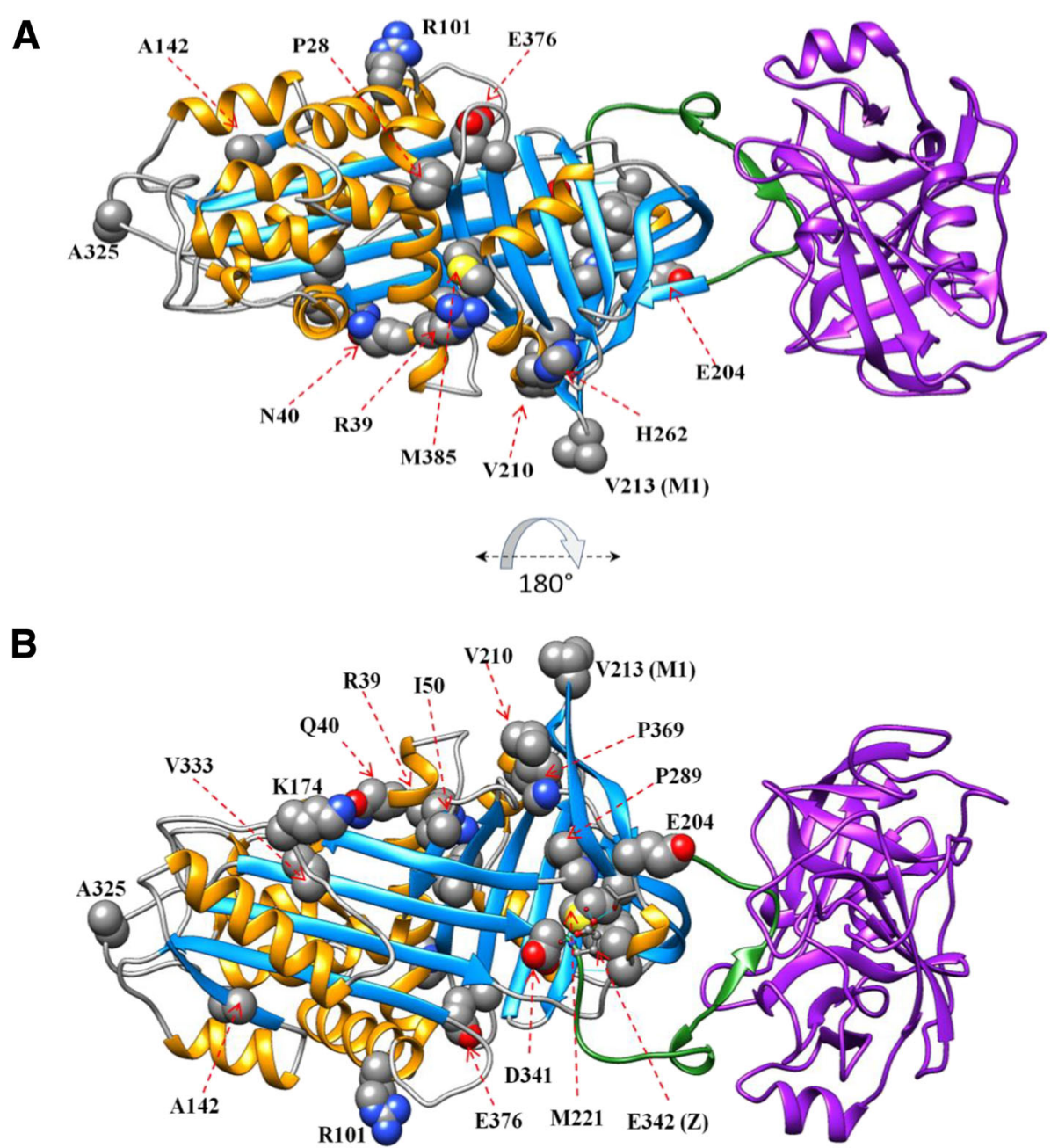

Fig. 1 Structure of AAT indicating the location of missense residues. The AAT protein (PDB code 1OPH) is shown in ribbon representation coloring according to secondary structural elements (alpha helices shown in orange, beta strands shown in light blue), and the position of missense changes showing the wildtype residue in sphere representation and labeled with the residue name and position. The purple ribbon protein is trypsinogen. The stretch of amino acids that comprise the reactive center loop are shown in green ribbon representation. $A=$ front view; $B=$ rear view (rotated 180 degrees about the $x$-axis). AAT, Alpha 1 Antitrypsin

$$
\mathrm{TNR}=\frac{\mathrm{TN}}{\mathrm{TN}+\mathrm{FP}}
$$

Positive predictive value (PPV): The proportion of predicted positives that are predicted correctly.

$$
\mathrm{PPV}=\frac{\mathrm{TP}}{\mathrm{TP}+\mathrm{FP}}
$$

Negative predictive value (NPV): The proportion of predicted negatives that are predicted correctly.

$$
\mathrm{NPV}=\frac{\mathrm{TN}}{\mathrm{TN}+\mathrm{FN}}
$$

Total accuracy (ACC): The proportion of true results (both true positives and true negatives) among the total number of experimental cases.

$$
\mathrm{ACC}=\frac{(\mathrm{TP}+\mathrm{TN})}{(\mathrm{P}+\mathrm{N})}=\frac{(\mathrm{TP}+\mathrm{TN})}{(\mathrm{TP}+\mathrm{FN})+(\mathrm{FP}+\mathrm{TN})}
$$

Balanced accuracy (BACC): This is the average of true positive rate (TPR) and true negative rate (TNR).

$$
\mathrm{BACC}=\frac{1}{2}(\mathrm{TPR}+\mathrm{TNR})
$$

\section{Results}

Patient characteristics

Patient characteristics $(n=23)$, including AATD phenotype, AAT level, and sequencing findings, are displayed in Table 1 . The male:female ratio was approximately $1: 1$, the age range was 34-87 years, and AAT levels ranged from $2 \mathrm{mg} / \mathrm{dL}$ to $160 \mathrm{mg} / \mathrm{dL}$. 


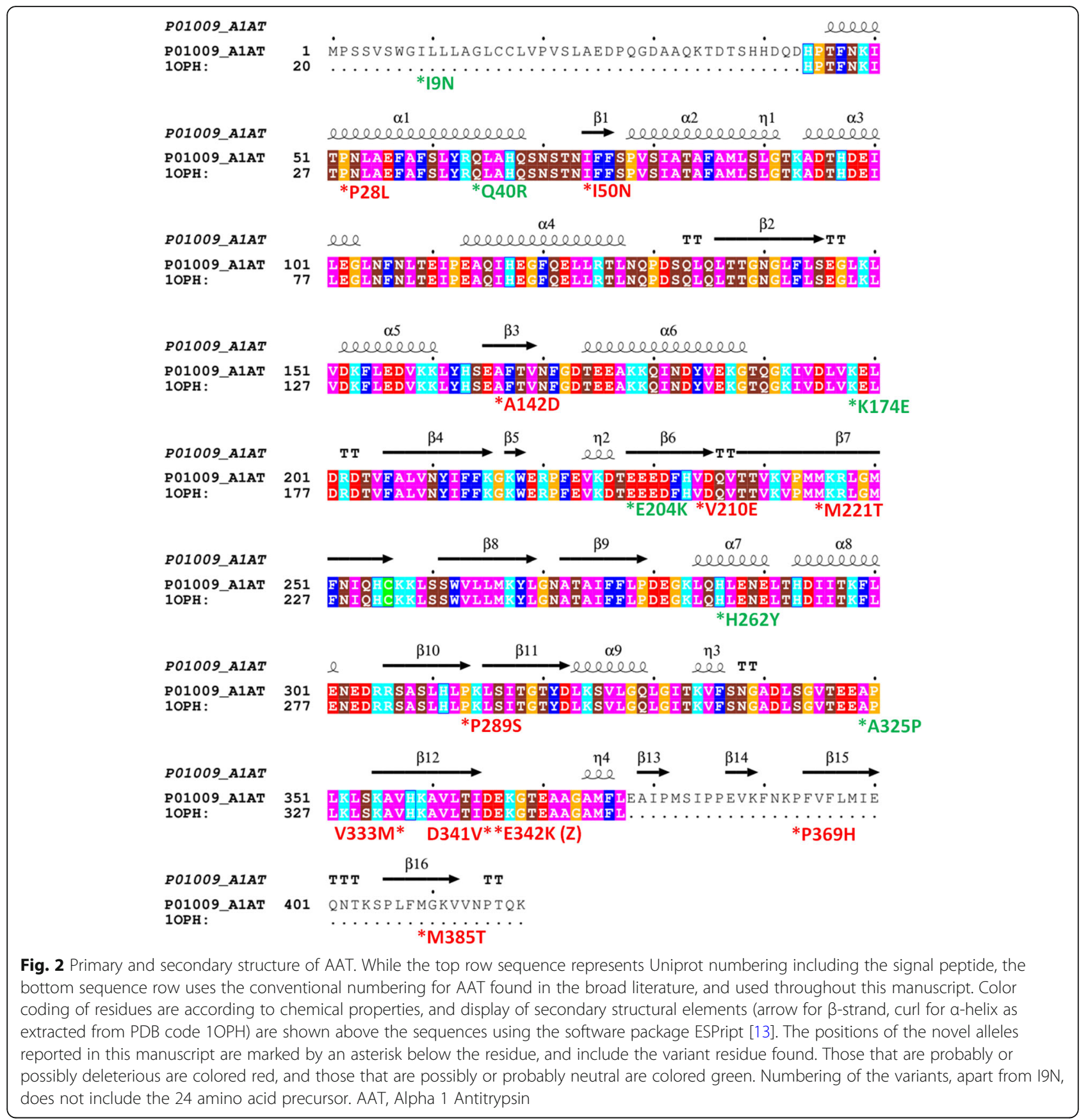

Clinical information on disease presentation was available from the four patients referred via Lewis Katz School of Medicine: patient CA97 presented with a cerebral aneurysm, patient 1144 presented with evidence of emphysema, and patient 4668 presented with chronic inflammatory demyelinating polyneuropathy (CIDP) in addition to emphysema. In addition, patient 76430 presented with severe emphysema/COPD and bronchiectasis. Detailed clinical descriptions of these patients will be reported separately. The remaining patients were referred to the $\mathrm{DNA}_{1}$ Advanced Alpha-1 Screening ${ }^{\text {Tw }}$ program by the treating physician due to clinical presentation or symptoms potentially indicative of AATD; i.e., COPD, asthma, emphysema, panniculitis, cerebral aneurysm, or liver disease.

\section{SERPINA1 mutations}

In this patient cohort, NGS DNA analysis identified 21 separate rare/novel variants. All amino acid changes are reported without the 24 amino acid precursor unless otherwise stated. The following variant types were identified: splice variants $(n=2)$, base pair deletions $(n=1)$, base pair changes resulting in a stop codon $(n=2$; one 


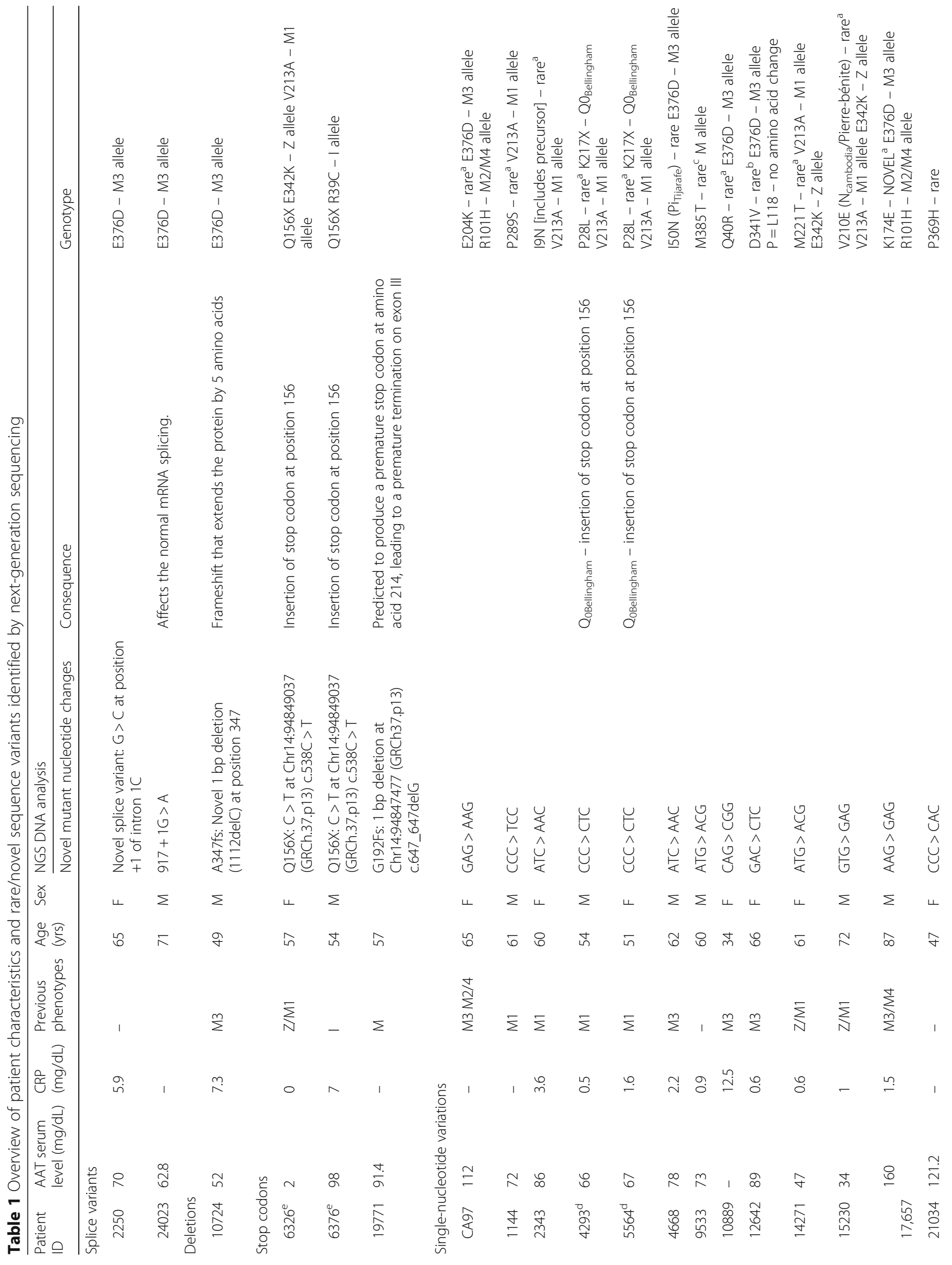




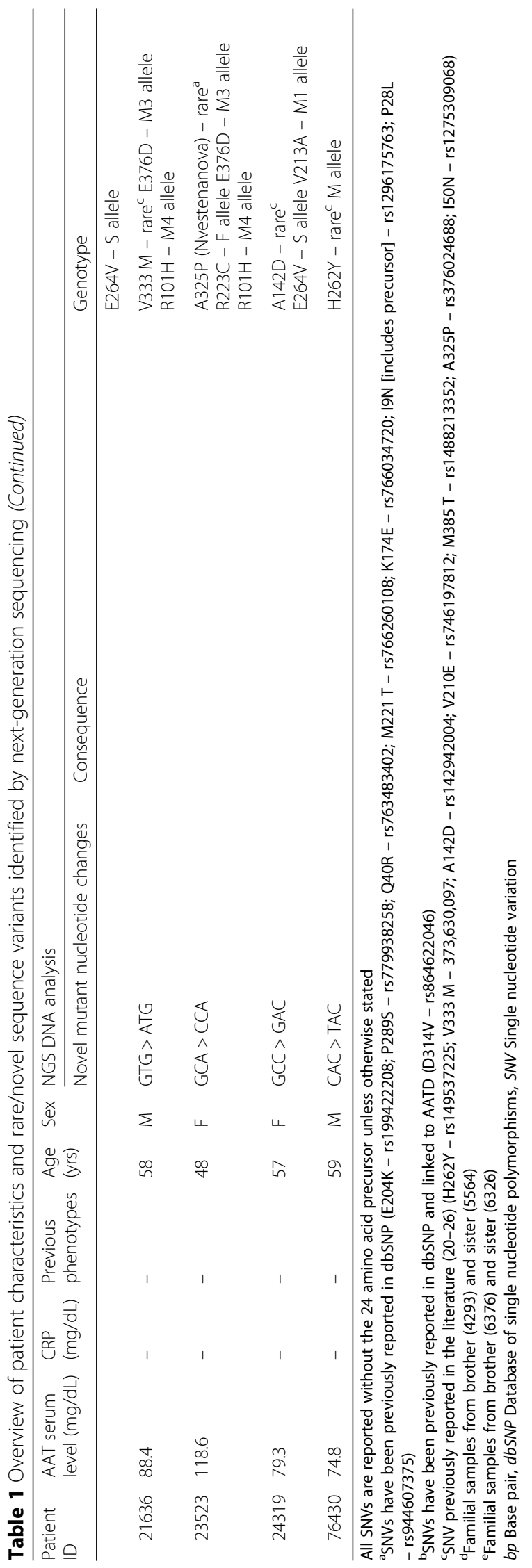


stop codon was found in two patients), and SNVs ( $n=$ 16; one novel SNV [P28L] was found in two patients) (Table 1).

\section{Splice variants}

A novel splice variant $(\mathrm{G}>\mathrm{C})$ was discovered at position +1 of intron $1 C$. The mutation occurred in a patient with no other SERPINA1 variants but with a low AAT level of $70 \mathrm{mg} / \mathrm{dL}$. A further splice variant $(6326 \mathrm{c} .917+$ $1 G>A)$, which resulted in an even lower AAT level of $62.6 \mathrm{mg} / \mathrm{dL}$, was discovered in patient 24023.

\section{Base pair deletions}

A single base-pair deletion was observed in patient 10724, with a low AAT level of $52 \mathrm{mg} / \mathrm{dL}$. The base pair deletion added 5 heterologous amino acids beyond position 347 before a stop codon, as well as truncating the remainder of the protein.

\section{Stop codons}

Sequence variants in two siblings (patients 6326 [female] and 6376 [male]), resulted in the insertion of a stop codon at position 156 (stop codon in normal AAT is position 418). Both had additional, well known pathogenic alleles: E342K (Z allele; patient 6326) and R39C (I allele; patient 6376). Combination with the $Z$ allele in patient 6326 resulted in extremely low serum AAT levels ( $2 \mathrm{mg} / \mathrm{dL})$. A further patient (ID 19771) was found to have a premature stop codon at amino acid 214, which resulted in an AAT level of $91.4 \mathrm{mg} / \mathrm{dL}$.

\section{SNVS}

Of the 16 rare/novel SNVs found in this investigation, two (found in patients 14271 and 15230) were heterozygous for the known pathogenic $\mathrm{Z}$ allele. In addition, two patients (21034 and 24319) were heterozygous for the known pathogenic S allele, and a further patient (23523) was heterozygous for the known pathogenic $\mathrm{F}$ allele. One novel SNV occurred twice in siblings (patients 4293 [male] and 5564 [female]) in combination with the known, rare, pathogenic Qobellingham variant. The remaining novel SNVs $(n=10)$ were heterozygous with the normal $M$ allele or $M$ subtypes (M1, M2, M2/4, etc.), which are secreted in similar concentrations and are comparable in function to the wildtype protein.

\section{Computational analysis of SNVs}

Computational predictions are presented in Table 2. Overall, the agreement between the SVM analysis and the additional computational predictors (FoldX and PolyPhen-2) was strong for all but two SNVs. Exceptions were the Q40R (patient 10889) and H262Y (patient 76430) sequence variants - both were associated with moderate deleterious scores by SVM (0.6589 and 0.6708, respectively), but the sequence variants were not predicted to destabilize the protein (i.e., they had small negative $\Delta \Delta G$ scores that indicate minor stabilization) and were predicted as benign by Polyphen- 2 .

\section{Probably deleterious variants}

Eight sequence variants were classified as probably deleterious (i.e., all three predictors registered a deleterious score). Two patients (14271 and 15230) were found to have novel mutations, M221T and V210E, respectively, in combination with the $\mathrm{Z}$ allele. Computational analyses strongly suggested that both novel sequence variants were deleterious. The AAT levels found in these samples (47 and $34 \mathrm{mg} / \mathrm{dL}$, respectively) were lower than would be expected for an individual with the PI*MZ genotype [66-100 mg/dL] [8] and were around the range of an individual homozygous for the $\mathrm{Z}$ allele [20-45 mg/dL] [1]. Additionally, two siblings (patients 4293 and 5564) presented with low AAT levels and a highly unusual genotype - the known pathogenic rare mutation $\mathrm{Q} 0_{\text {bellingham }}$ was accompanied by the novel mutation P28L, which all three computational analyses predicted to be damaging.

Most of the remaining rare/novel SNVs that the computational analyses predicted to be probably pathogenic were heterozygous with normal alleles. The presence of the P289S (patient 1144), I50N (patient 4668), D341V (patient 12642), or A142D (patient 24319) sequence variants appeared to result in AAT levels ranging from 72 to $89 \mathrm{mg} / \mathrm{dL}$ - levels that are often associated with PI*MZ individuals. Moreover, patients 1144 and 4668, who presented with the P289S and I50N mutations, respectively, were recorded as having lung disease.

There was only one exception from the general agreement between computational predictions and AAT serum levels - the $\mathrm{P} 369 \mathrm{H}$ mutation, observed in patient 21034. All three computational analyses predicted the mutation to be highly deleterious; however, the AAT serum level was normal $(121.2 \mathrm{mg} / \mathrm{dL})$. This may have been due to the presence of an inflammatory state at the time of sampling; unfortunately, a CRP value was not available for this patient.

\section{Possibly deleterious variants}

Two sequence variants were classified as possibly deleterious (i.e., two of three predictors registered a deleterious score). The variant M385 T (patient 9533) was found in combination with a wildtype allele. The M385 $\mathrm{T}$ variant is a good example of how methods that focus on a variety of structural parameters for prediction may be more effective than those that more heavily weight sequence conservation. Both the SVM deleterious result and the FoldX prediction of a significant drop in stability make this variant likely deleterious. These predictions correspond to an AAT level of $73 \mathrm{mg} / \mathrm{dL}$, which was 


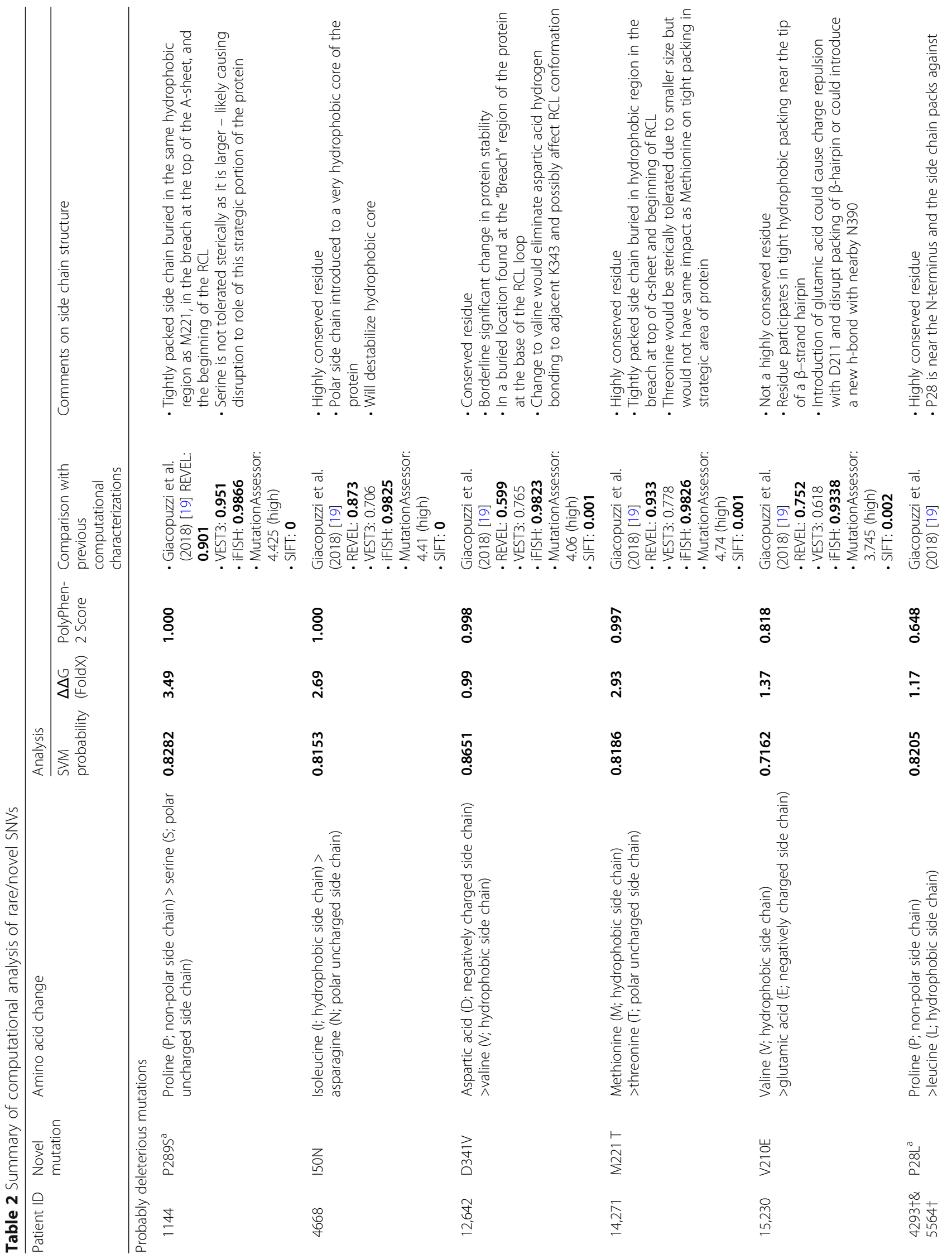


Kueppers et al. BMC Medical Genetics

(2019) 20:125

Page 10 of 19

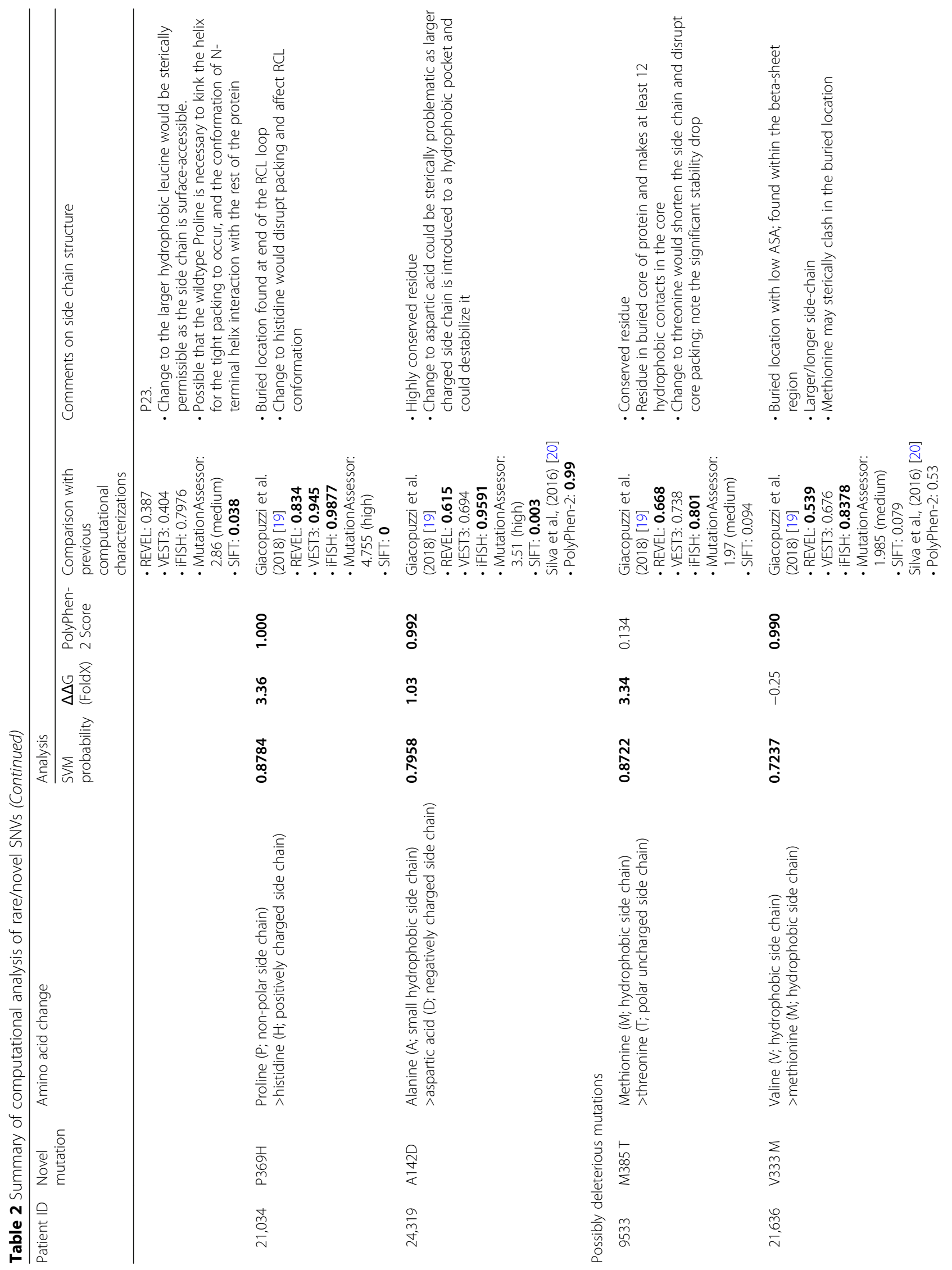




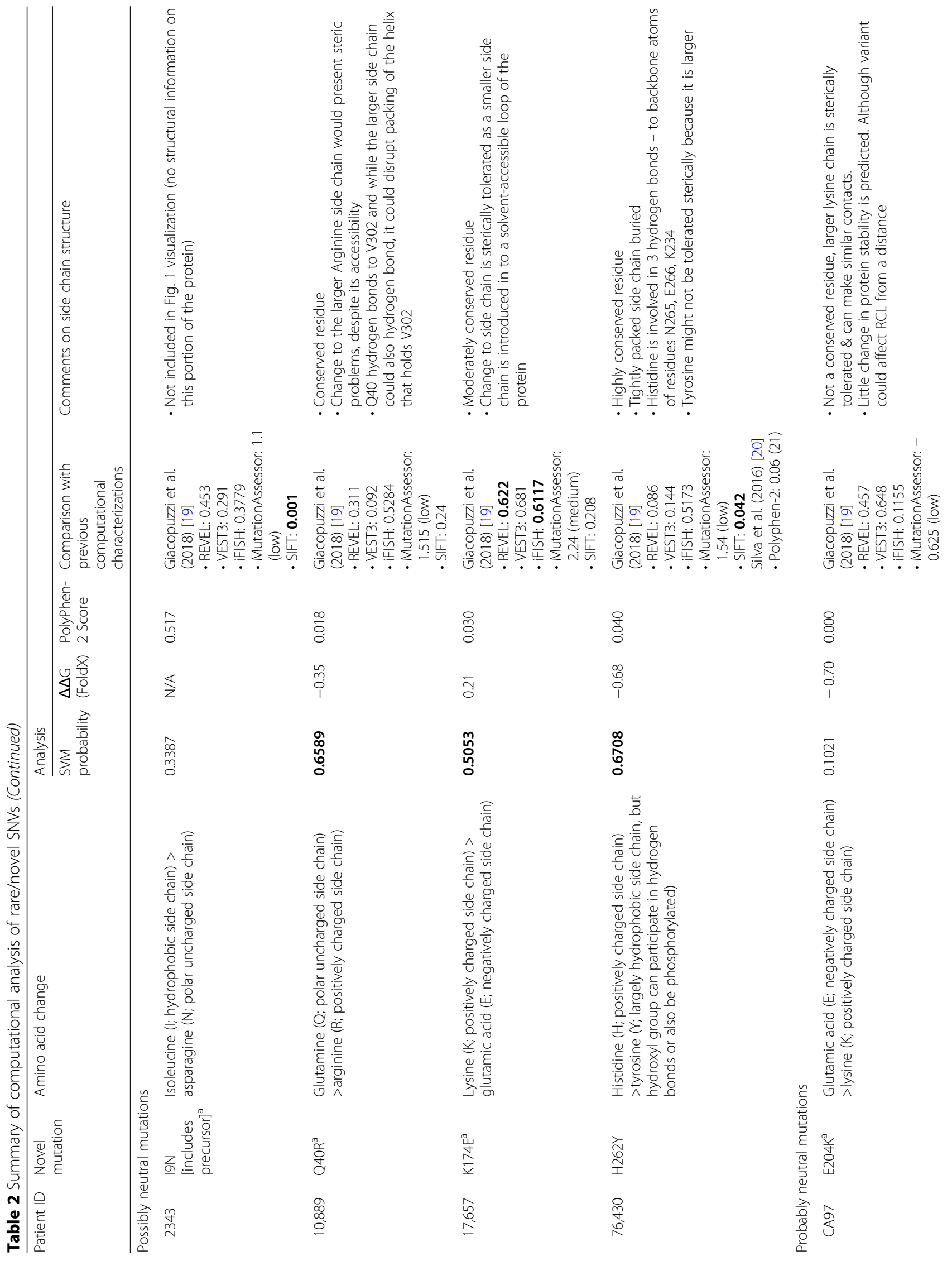


$\begin{array}{ll}\text { Kueppers et al. BMC Medical Genetics } & \text { (2019) 20:125 }\end{array}$

Page 12 of 19

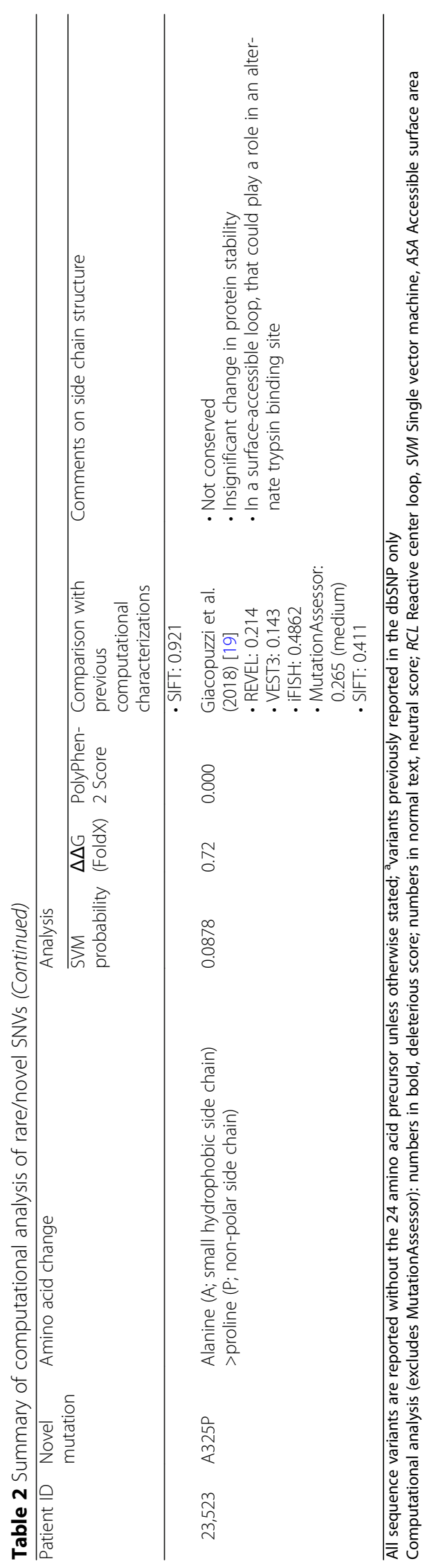


Table 3 Results of benchmarking analysis

\begin{tabular}{|c|c|c|c|c|c|}
\hline SERPINA1 mutation & Species & Sequence Identity (\%) & SVM probability & $\Delta \Delta \mathrm{G}($ Fold $X)$ & PolyPhen-2 Score \\
\hline \multicolumn{6}{|c|}{ Pathogenic sequence variants (ClinVar) } \\
\hline P369L & Homo sapiens & 100 & 0.8496 & 1.510 & 1.0000 \\
\hline P369S & Homo sapiens & 100 & 0.8353 & 4.410 & 1.0000 \\
\hline Р369T & Homo sapiens & 100 & 0.8599 & 2.810 & 1.0000 \\
\hline M358R & Homo sapiens & 100 & 0.7853 & 0.431 & 0.0190 \\
\hline E342K & Homo sapiens & 100 & 0.8413 & 2.090 & 1.0000 \\
\hline E264V & Homo sapiens & 100 & 0.8619 & 1.660 & 1.0000 \\
\hline D256V & Homo sapiens & 100 & 0.8708 & 1.950 & 0.9850 \\
\hline G225R & Homo sapiens & 100 & 0.8975 & 5.160 & 0.9820 \\
\hline R223C & Homo sapiens & 100 & 0.8954 & -0.350 & 0.9950 \\
\hline $192 \mathrm{~N}$ & Homo sapiens & 100 & 0.8215 & 3.600 & 1.0000 \\
\hline G67E & Homo sapiens & 100 & 0.8680 & 26.290 & 1.0000 \\
\hline S53F & Homo sapiens & 100 & 0.8687 & 19.860 & 1.0000 \\
\hline L41P & Homo sapiens & 100 & 0.7585 & 3.240 & 0.6010 \\
\hline R39C & Homo sapiens & 100 & 0.8672 & 2.110 & 1.0000 \\
\hline A336T & Homo sapiens & 100 & 0.8479 & 3.450 & 1.0000 \\
\hline G115S & Homo sapiens & 100 & 0.7826 & 1.610 & 0.9990 \\
\hline F52S & Homo sapiens & 100 & 0.7900 & 6.020 & 1.0000 \\
\hline \multicolumn{6}{|c|}{ Benign sequence variants (ClinVar) } \\
\hline E376D & Homo sapiens & N/A & 0.2991 & 1.850 & 0 \\
\hline E363K & Homo sapiens & N/A & 0.4172 & -0.900 & 0.11 \\
\hline A284S & Homo sapiens & N/A & 0.3445 & -0.240 & 0.139 \\
\hline V213A & Homo sapiens & N/A & 0.1161 & -0.100 & 0 \\
\hline $\mathrm{R} 101 \mathrm{H}$ & Homo sapiens & N/A & 0.0576 & -0.533 & 0 \\
\hline \multicolumn{6}{|c|}{ Benign sequence variants (Primate neutral variants) } \\
\hline P21Q & Hylobates sp. ECACC & 95 & 0.363 & 0.72 & 0.014 \\
\hline F23L & Papio anubis & 92 & 0.3762 & 0.38 & 0 \\
\hline T27A & Gorilla gorilla & 98 & 0.4118 & 1.34 & 0 \\
\hline N29K & Hylobates sp. ECACC & 95 & 0.4365 & -0.63 & 0.178 \\
\hline N29S & Papio anubis & 92 & 0.0839 & 0.13 & 0 \\
\hline T48S & Hylobates sp. ECACC & 95 & 0.4991 & 0.51 & 0 \\
\hline I50V & Gorilla gorilla & 98 & 0.6687 & 0.96 & 0.767 \\
\hline D74S & Chlorocebus sabaeus & 92 & 0.0588 & 0.38 & 0 \\
\hline $\mathrm{N} 81 \mathrm{H}$ & Pongo abelii & 96 & 0.462 & 0.27 & 0.007 \\
\hline $192 \mathrm{~V}$ & Pongo abelii; Hylobates sp. ECACC & $96 ; 95$ & 0.7931 & 1.06 & 0.006 \\
\hline Q105K & Papio anubis & 92 & 0.2178 & -0.5 & 0.001 \\
\hline N116S & Gorilla gorilla & 98 & 0.751 & 1.9 & 0.311 \\
\hline $\mathrm{K} 136 \mathrm{~N}$ & Papio anubis & 92 & 0.4292 & 0.07 & 0 \\
\hline E141D & Pongo abelii & 96 & 0.4052 & 0.77 & 0.002 \\
\hline G148E & Chlorocebus sabaeus & 92 & 0.219 & -0.46 & 0 \\
\hline D159N & Papio anubis & 92 & 0.165 & -1.21 & 0 \\
\hline Q212E & Pongo abelii & 96 & 0.2568 & 0.35 & 0 \\
\hline V213A & Hylobates sp. ECACC & 95 & 0.1161 & -0.1 & 0 \\
\hline Q230Y & Papio anubis & 92 & 0.0803 & 0.49 & 0 \\
\hline
\end{tabular}


Table 3 Results of benchmarking analysis (Continued)

\begin{tabular}{|c|c|c|c|c|c|}
\hline SERPINA1 mutation & Species & Sequence Identity (\%) & SVM probability & $\Delta \Delta \mathrm{G}($ Fold $\mathrm{X})$ & PolyPhen-2 Score \\
\hline Q230H & Hylobates sp. ECACC & 95 & 0.0545 & 0.41 & 0 \\
\hline K233E & Papio anubis & 92 & 0.0787 & 0.58 & 0 \\
\hline $\mathrm{D} 270 \mathrm{E}$ & Hylobates sp. ECACC & 95 & 0.2543 & -0.03 & 0 \\
\hline $1271 \mathrm{~V}$ & Gorilla gorilla & 98 & 0.1015 & 0.52 & 0 \\
\hline D280N & Pongo abelii & 96 & 0.1407 & 0.82 & 0 \\
\hline S285N & Chlorocebus sabaeus & 92 & 0.0554 & -1.6 & 0 \\
\hline S292A & Chlorocebus sabaeus & 92 & 0.3206 & -0.14 & 0.003 \\
\hline S301R & Pongo abelii & 96 & 0.1328 & -0.54 & 0.011 \\
\hline S301T & Hylobates sp. ECACC & 95 & 0.0724 & 0 & 0 \\
\hline S313G & Hylobates sp. ECACC & 95 & 0.606 & 0.63 & 0.05 \\
\hline E324D & Chlorocebus sabaeus & 92 & 0.0978 & 0.62 & 0 \\
\hline A332V & Homo sapiens & 99 & 0.1255 & 1.88 & 0.99 \\
\hline I360V & Gorilla gorilla & 98 & 0.1547 & 0.73 & 0 \\
\hline $\mathrm{L} 383 \mathrm{H}$ & Hylobates sp. ECACC & 95 & 0.8834 & 3.08 & 1 \\
\hline M385I & Papio anubis & 92 & 0.7686 & 3.15 & 0.001 \\
\hline M385 V & Pan troglodytes; Pongo abelii & $99 ; 96$ & 0.7566 & 2.44 & 0.001 \\
\hline
\end{tabular}

All variants are reported without the 24 amino acid precursor unless otherwise stated

Scores in bold, pathogenic; scores in italics, possibly pathogenic; scores in normal text, benign. SVM Support vector machine

likely to be mainly contributed by the wildtype (normal) allele in this patient. While Polyphen-2 predicted the M385 T variant to be benign, the added structural information considered by both the SVM and FoldX predictors contributed to a greater sensitivity to detect this variant as deleterious. Lastly in this category, the variant V333 M (patient 21636) was found in combination with M3 and M4 alleles and a serum level of $88.4 \mathrm{mg} / \mathrm{dL}$. The SVM and Polyphen-2 predictions were deleterious, while the FoldX score predicted no destabilization of the protein.

\section{Possibly neutral variants}

Four sequence variants were predicted to be possibly neutral (only one of the three predictors scored as deleterious). The $19 \mathrm{~N}$ (includes precursor) found in patient 2343 was classified as possibly neutral as the SVM prediction (0.3387) was below the deleterious threshold, accompanied by a borderline pathogenic score (0.517) from PolyPhen-2, and a moderate AAT level of $86 \mathrm{mg} /$ $\mathrm{dL}$. As this mutation is in the cleaved precursor region of AAT and as there are no coordinates for this residue in the protein structure, a Gibbs free energy change cannot be calculated. The Q40R variant had an SVM score of 0.6589 (a moderately deleterious result), but was not predicted to destabilize the protein, and scored benign by Polyphen-2. This variant was accompanied by a second M3 allele, and serum AAT levels were not obtained for the patient (10889). A novel mutation from patient 17657 (K174E) was predicted by SVM to have a borderline deleterious score of 0.5053 ; however, the score had \pm 0.036 standard deviation and could thus potentially be below the threshold for deleterious. This was accompanied by benign predictions by FoldX and Polyphen-2, and was associated with normal AAT levels $(160 \mathrm{mg} / \mathrm{dL})$. The H262Y variant (patient 76430 ) was associated with a moderate deleterious prediction by SVM (0.6708), but was not predicted to destabilize the protein, and was predicted benign by Polyphen- 2 . Nonetheless, the low serum AAT level of $74.8 \mathrm{mg} / \mathrm{dL}$ found in this patient accompanied by the presence of lung disease are suggestive of deleterious effects.

\section{Probably neutral variants}

In this last category, two variants were predicted to be probably neutral (i.e., none of the three predictors scored as deleterious). One mutation in a non-conserved residue (E204K) found in patient CA97 was predicted to be neutral by all predictors - this is supported by the normal AAT level found in this patient $(112 \mathrm{mg} / \mathrm{dL})$. Similarly, the A325P mutation (patient 23523) was accompanied by a normal AAT level of $118.6 \mathrm{mg} / \mathrm{dL}$, with agreement among the three predictions that the mutation was neutral.

\section{Benchmarking of SVM predictions}

Table 3 provides the SVM predictions for each of the benign and pathogenic variants included in the benchmarking analysis, with comparisons to PolyPhen2 and FoldX predictions. For the pathogenic variant set, 17/17 $(100 \%)$ were predicted to be deleterious by SVM, and correspondingly $16 / 17$ variants $(94.1 \%)$ were predicted 
to have negative effects on stability of the protein by FoldX. PolyPhen 2 predicted 16/17 (94.1\%) to be pathogenic. For the benign ClinVar set, 5/5 variants (100\%) were predicted by both the SVM and PolyPhen- 2 to be benign, and correspondingly $4 / 5$ were predicted by FoldX to slightly improve protein stability (negative values indicate better predicted stability with the variant). In the alternative benign primate dataset, $28 / 35$ variants $(80 \%)$ were predicted to be benign by the SVM, compared with $32 / 35$ predicted $(91.4 \%)$ predicted to be benign by PolyPhen- 2 . The statistical parameters calculated suggest that the accuracy of both SVM and PolyPhen-2 predictors are broadly similar (Table 4). While the sample sizes of this benchmark set are not sufficient for a comprehensive comparison of the SVM to Polyphen-2 or other predictors, overall the benchmark testing on these variants of known effect on SERPINA1 function validates the strength and accuracy of the SVM and Polyphen-2 for predictions on novel variants presented in this work.

\section{Discussion}

Through the $\mathrm{DNA}_{1}$ Advanced Alpha-1 Screening ${ }^{\mathrm{Tw}}$ program, we have begun to encounter large numbers of novel sequence variants of the SERPINA1 gene, as evidenced by the data we have presented. The present study is supportive of several earlier investigations that have uncovered previously uncharacterized and potentially pathogenic sequence variants of SERPINA1 [7, 9, $12,19,21]$. There is a growing body of evidence to suggest that novel sequence variants may be more clinically impactful than previously thought, with some reported to be associated with early onset COPD [9].

Using NGS, we identified 21 rare/novel sequence variants of the SERPINA1 gene in patients suspected of having AATD. Most of the variants $(n=16)$ were SNVs. In addition, two base pair changes resulting in stop codon insertions, one base pair deletion, and two splice variants were discovered. All of the SNVs were previously recorded in the National Center for Biotechnology Information's database of single nucleotide polymorphisms (dbSNP) and/or in the literature [19, 20, 22-26] (Table 1). The $\mathrm{I} 50 \mathrm{~N}$ variant $\left(\mathrm{Pi}_{\text {Tijarafe }}\right)$ was previously confirmed as pathogenic in an vitro cell model, and was associated with similar AAT expression to the $\mathrm{Z}$ variant [26]. Nonetheless, to the best of our knowledge, this is the first study to describe seven variants (E204K, P289S, Q40R, M221T, K174E, I9N [includes precursor] and P28L) alongside additional patient data. However, despite the availability of other data such as AAT levels, determining whether these variants are clinically relevant is challenging. We therefore sought to evaluate the utility of computational modeling to provide supporting evidence, in addition to observed AAT serum levels, of the pathogenicity of rare SNVs. We note that computational methods predict the effects of missense variants on either protein function (SVM, and machine learning approaches) or the inherent stability of the tertiary/quaternary structure of a protein (FoldX). However, this may not always correspond with clinical parameters, such as secreted protein serum levels, or the degree of pathogenicity in a particular organ.

The majority of the sequence variants identified in our cohort were predicted to be deleterious by computational methods. Only two variants were predicted to be probably neutral by all three computational techniques. Of the rare variants previously reported in the dbSNP only (E204K, P289S, Q40R, M221T, K174E, I9N [includes precursor] and $\mathrm{P} 28 \mathrm{~L}$ ), the probably deleterious variants were predicted to be, P289S, M221T, and P28L, and were accompanied by low AAT levels. In particular, the P289S variant was found in a 61-year-old patient with advanced emphysema, supporting the pathogenicity of this variant. The remaining variants were predicted to be neutral or possibly neutral, and were accompanied by normal or low-normal AAT levels (although no AAT level was reported with the Q40R variant), and are less likely be clinically relevant. Although there is some evidence of a relationship between AAT variants and cerebral aneurisms [27], we do not have sufficient evidence to conclude a causal relationship between the clinical presentation in patient (CA97) and the E204K variant. For the rare variants predicted to be probably deleterious or possibly deleterious, in line with previous reports, we observed that the majority of these cluster around functional domains of AAT [20]. The mechanism of pathogenicity for most of these sequence variants (I50N, P289S, M385T, M221T, D341V, V210E, P369H, V333M and A142D) is likely to be via disruption of the tightly packed hydrophobic core of the AAT protein, and some may in turn disrupt the adjacent reactive center loop (RCL; Fig. 3) that inhibits proteases. One possible mechanism is that substantial changes to the core of the protein could result in misfolding of the protein within hepatocytes, such that only small amounts of AAT would be released, resulting in reduced levels of AAT in the peripheral circulation. An alternative mechanism of pathogenicity might include missense changes that do

Table 4 Measurements of benchmarking predictions

\begin{tabular}{lllllll}
\hline & TPR (sensitivity) & TNR (specificity) & PPV & NPV & ACC & BACC \\
\hline SVM & 1.0 & 0.825 & 0.720 & 1.0 & 0.879 & 0.913 \\
Polyphen2 & 0.944 & 0.925 & 0.85 & 0.974 & 0.931 & 0.935 \\
\hline
\end{tabular}

TNR True negative rate, TPR True positive rate, PPV Positive predictive value, NPV Negative predictive value, ACC Total accuracy, BACC Balanced accuracy 


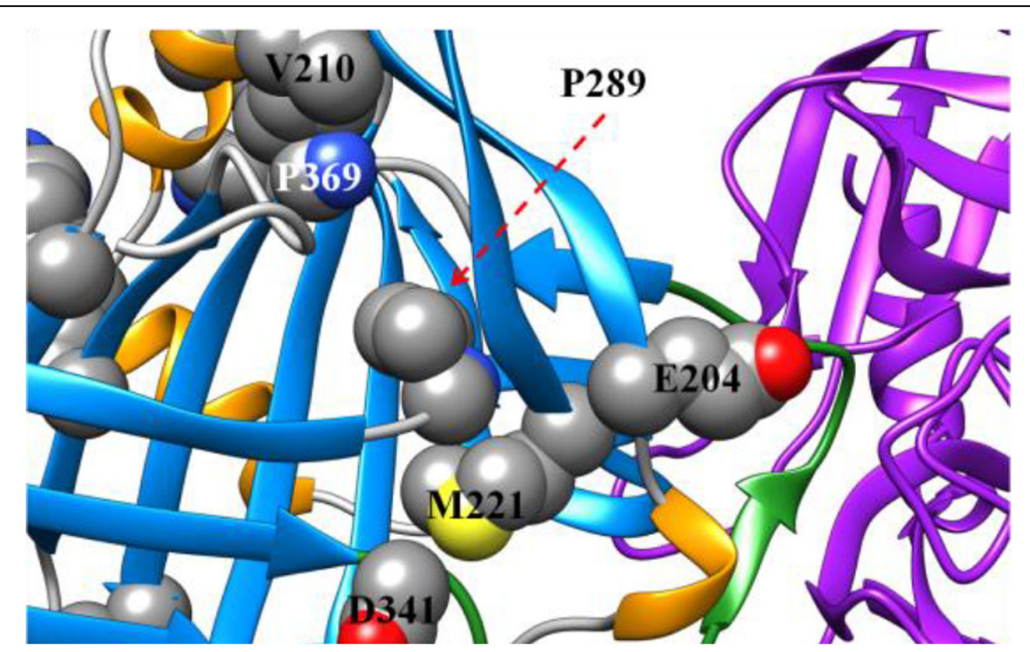

Fig. 3 Structure of AAT zoomed in on locations of interest. Presented are some of the missense residues predicted to negatively affect the stability of the folded protein. Several of these missense changes are in the tightly packed core of the protein, such as the P289S variant packed tightly near the M221T variant location

not affect AAT folding and result in normal levels detected in serum, but have a deleterious effect on conformational changes required for sheet opening or protein-protein interactions necessary for inhibition of neutrophil elastase.

As expected, very low blood levels of AAT were found in heterozygotes for known deficiency alleles and new mutations. Two patients (12230 and 15230) in this study had very low AAT levels around the range associated with a PI"ZZ individual [20-45 mg/dL] [1], and novel pathogenic variants in combination with the $\mathrm{Z}$ allele. $\mathrm{Pa}$ tients such as these would be strong candidates for AAT therapy if they presented with airflow obstruction and significant emphysema [28]. There are more than 6 million individuals in the United States alone with the $\mathrm{PI}^{*} \mathrm{MZ}$ genotype [5]. As shown by this study, it is possible that numerous other patients may be undiagnosed compound heterozygotes with rare/novel sequence variants not detectable by IEF or targeted genotyping. The concept of cumulative deleterious effects in compound heterozygotes has previously been described for the $\mathrm{PI} F \mathrm{FZ}$ genotype [29]. The F allele is associated with normal AAT levels but reduced AAT functionality, while low circulating levels are observed in Z patients [29]. All AAT secreted by $\mathrm{PI}^{*} \mathrm{FF}$ homozygotes has reduced functionality and these individuals have been shown to be at increased risk of lung damage caused by uninhibited elastase [29]. In PI*FZ heterozygotes, functionality and circulating levels are both reduced, resulting in an increased risk of emphysema compared with PI*FF patients [29].

Most novel sequence variants within our cohort were heterozygous with normal variants; it is therefore difficult to fully assess the impact of these variants on serum
AAT levels and risk of emphysema. For known variants the disease risk is well known. For example, individuals with the PI*MZ genotype have a greater degree of airflow obstruction than PI*MM individuals with comparable smoke exposure, and ever-smoking PI*MZ individuals have an increased risk of developing COPD [30]. However, the longitudinal disease-risk associated with rare alleles is unknown and AAT levels, although indicative of severity, are not conclusive. As the majority of these rare/novel variants will probably have different mechanisms of pathogenicity, it is possible that the disease risk is different to that of common heterozygotes and is specific for each variant. Further biochemical and clinical characterization is needed to fully understand how these sequence variants contribute to lung disease.

AATD is usually associated with single amino acid substitutions/deletions leading to subtle structural changes to the AAT protein; however, this study also identified splice variants, stop codons, and large deletions in SERPINA1. The potential contribution of these sequence variants to AATD should not be underestimated, especially when occurring in combination with damaging structural mutations. For example, in patient 6326, insertion of a stop codon at position 156 in combination with the $\mathrm{Z}$ mutation resulted in a severe reduction in antigenic AAT levels $(2 \mathrm{mg} / \mathrm{dL})$. This effect was not apparent in this patient's sibling (patient 6376), whose AAT level was $98 \mathrm{mg} / \mathrm{dL}$. Patient 6376 is heterozygous for the above mentioned stop codon and the PI*I (R39C) allele - the PI*I mutation gives rise to a misfolded AAT protein, which is present in peripheral blood at near-normal concentrations [31]. This further demonstrates that rare and novel sequence variants can become more clinically relevant in combination with common deficiency alleles. 
For patients with rare/novel mutations, apart from instances where the variants are deletions or null variants, it can be difficult to determine the impact of sequence variants and if treatment with exogenous AAT is necessary. This study has demonstrated that computational analyses may be useful in understanding the potential impact of novel mutations. The three predictive computational methods presented were generally in agreement and in most cases related to the observed AAT levels. In particular, we found that the enhanced structural information that contributes to the SVM predictions may confer a greater sensitivity to deleterious variants, making it suited for clinical genetics applications. The benchmarking analysis provides a strong validation for the balanced accuracy of the SVM predictions and supports its use in predicting the effects of the novel variants described in the current work. In addition, there was good agreement between results of the present analysis and previous studies [19, 20] (Table 2). One exception to the general agreement between this and previous studies may be $\mathrm{P} 28 \mathrm{~L}$, with other computational measures suggesting that it is of intermediate pathogenicity. However, it is notable that the number of previously reported deleterious scores generally mirror that of those reported in the present study through the categories of probably deleterious, possibly deleterious, possibly neutral and probably neutral utilized in the present study. In particular, in the probably neutral section, no deleterious scores are presented from this analysis or previous reports.

Some important limitations of this study should be mentioned. This observational study was not controlled, i.e., there were no formal inclusion and exclusion criteria and no control group, and data were collected from a small $(N=23)$ patient population. In addition, genetic and non-genetic factors - not related to the AAT sequence variants reported here - may have contributed to the development of COPD. However, these factors are beyond the scope of the current report. Furthermore, computational modeling of missense variants only predicts if a substitution is deleterious to protein function or stability. We do not know the exact mechanisms by which these substitutions lead to either reduced AAT levels or weakened elastase-inhibiting activity. Furthermore, it should be noted that a host of different modeling software are available, and each may produce different results for a particular mutation, as demonstrated by Giacopuzzi et al. (Table 2). It was outside the scope of the present study to assess a wide range of modeling techniques, as a further aim of the study was to relate the computational scores to clinical parameters. However, Giacopuzzi et al. raise an important point, in that no individual computational method is infallible, and in an ideal situation, more than one technique should be consulted in the clinical decision-making process. In addition, computational predictions may be inconsistent with findings of experimental characterization; therefore, ultimately, detailed biochemical functional analysis of the protein is required to validate the findings of computational analyses. In addition, clinical information on patient presentation is required in order to obtain a full picture of the patient's individual disease risk.

Despite the above limitations, this study demonstrates that there are numerous potentially pathogenic novel variants beyond those commonly associated with AATD. Due to the progressive and irreversible destruction of lung tissue seen in severe AATD, early and accurate diagnosis is crucial to prevent further loss of lung tissue. Data from the RAPID/RAPID Extension trials has demonstrated that while treatment with AAT can slow the loss of lung tissue, tissue lost prior to commencing treatment cannot be regained $[32,33]$. This is compounded by the fact that patients often experience long delays before receiving an accurate diagnosis [34], partly due to a lack of specialized testing. Early diagnosis also enables patients to implement lifestyle changes such as smoking cessation and avoidance of passive smoke. However, identifying rare/novel variants can be difficult, and this task may be impossible by traditional methods such as protein phenotyping via IEF [10].

The increasing availability of commercial DNA testing is helping to improve diagnosis of patients with AATD and rare genotypes [35]. However, many current approaches do not incorporate sequencing, and are unable to detect potentially pathogenic rare/novel variants that may lead to development of AATD. The need for faster screening and diagnosis of AATD has led to the development of the $\mathrm{DNA}_{1}$ Advanced Alpha-1 Screening ${ }^{\mathrm{TM}}$ Program. $\mathrm{DNA}_{1}$ testing incorporates AAT levels, C-reactive protein serum levels, targeted genotyping (including the F and I alleles), and IEF, and reflexes to NGS when these methods prove insufficient. Our results support the proposal by Graham et al, who recommended that individuals with low serum levels and no resolution in targeted tests should be subjected to full-gene sequencing [12].

\section{Conclusions}

Advancements in DNA sequencing technology continue to reveal numerous rare/novel sequence variants in the SERPINA1 gene. Many of these variants may be pathogenic and causative factors in the development of AATD. Computational modeling opens new dimensions of structural analysis, which can help to define the pathogenic nature of these variants more accurately. The computational analyses we present are straightforward to perform and can provide a valuable additional indication (in combination with serum levels and clinical presentation) of the pathogenicity of novel mutations. We expect that this added information will eventually lead to improved individualized therapy for patients with AATD. 


\section{Additional file}

Additional file 1: Protein modeling to assess the pathogenicity of rare variants of SERPINA1 in patients suspected of having Alpha 1 Antitrypsin Deficiency (DOCX $25 \mathrm{~kb})$

\section{Abbreviations}

AAT: Alpha 1 Antitrypsin; AATD: Alpha 1 Antitrypsin Deficiency; IEF: Isoelectric focusing; NE: Neutrophil elastase; NGS: Next-generation sequencing RCL: Reactive Center Loop; SNP : Single-nucleotide polymorphism; SNV: Single-nucleotide variation; SVM: Support vector machine

\section{Acknowledgements}

Medical writing assistance was provided by Steven Foster of Meridian HealthComms Ltd., Plumley, UK, funded by CSL Behring.

\section{Authors' contributions}

FK recruited certain patients and performed their clinical assessment; MA, QX, and RD performed the computational modeling analyses and assisted with the interpretation of the findings. CS and JK conducted all laboratory analyses and reviewed the manuscript. All authors participated in the writing of the manuscript, and reviewed and approved the manuscript for submission.

\section{Funding}

This research was funded by CSL Behring. Development of the DNA, Advanced Alpha-1 Screening ${ }^{\text {TM }}$ kit and the laboratory testing of the clinical samples were performed by Biocerna LLC, funded by CSL Behring. Medical writing assistance was funded by CSL Behring. Research of M.A., R.D., and Q.X. at the Fox Chase Cancer Center Molecular Modeling Facility is supported in part by National Institutes of Health grant CA 06927 and R35 GM122517. The role of CSL Behring in this study was to fund data collection activities and support writing assistance only; the National Institutes of Health grants supported aspects of the research presented here. The funders did not participate in the design of the study, data collection, analysis, interpretation of data, and manuscript preparation/writing.

\section{Availability of data and materials}

All sequencing data reported have been deposited within a publicly accessible database (NCBI BioProject; Accession: PRJNA547351; URL: https:// www.ncbi.nlm.nih.gov/bioproject/?term=PRJNA547351). All other available data are reported within this manuscript and its Additional file.

\section{Ethics approval and consent to participate}

Written informed consent for use of laboratory data for research purposes was provided by all patients included in this study. The study was approved by the Institutional Review Board of Temple University, Philadelphia, PA.

\section{Consent for publication}

No identifiable individual patient data are reported.

\section{Competing interests}

Development of the DNA1 Advanced Alpha-1 Screening ${ }^{\mathrm{TM}}$ kit and the laboratory testing of the clinical samples were performed by Biocerna LLC, funded by CSL Behring. F.K., M.D.A, Q.X., and R.L.D. have nothing to declare. J.K. is an employee of Biocerna. C.S. is the founder and CEO of Biocerna LLC.

\section{Author details}

'Department of Thoracic Medicine and Surgery, Lewis Katz School of Medicine, Temple University, Philadelphia, PA, USA. ${ }^{2}$ Molecular Therapeutics Program, Institute for Cancer Research, Fox Chase Cancer Center, Philadelphia, Pennsylvania, USA. ${ }^{3}$ Biocerna LLC, Fulton, Maryland, USA.

\section{Received: 27 November 2018 Accepted: 24 June 2019}

\section{Published online: 15 July 2019}

\section{References}

1. American Thoracic Society, European Respiratory Society. American Thoracic Society/European Respiratory Society statement: standards for the diagnosis and management of individuals with alpha-1 antitrypsin deficiency. Am J Respir Crit Care Med. 2003;168:818-900.

2. Stoller JK, Aboussouan LS. A review of alpha1-antitrypsin deficiency. Am J Respir Crit Care Med. 2012;185:246-59.

3. Gadek JE, Fells GA, Zimmerman RL, Rennard SI, Crystal RG. Antielastases of the human alveolar structures. Implications for the protease-antiprotease theory of emphysema. J Clin Invest. 1981;68:889-98.

4. Lomas DA. The selective advantage of alpha1-antitrypsin deficiency. Am J Respir Crit Care Med. 2006;173:1072-7.

5. Blanco I, de Serres FJ, Carcaba V, Lara B, Fernandez-Bustillo E. Alpha-1 antitrypsin deficiency $\mathrm{Pl}^{*} \mathrm{Z}$ and $\mathrm{Pl}^{*} \mathrm{~S}$ gene frequency distribution using on maps of the world by an inverse distance weighting (IDW) multivariate interpolation method. Hepat Mon. 2012;12:e7434.

6. Kueppers F. Chapter 12: chronic obstructive pulmonary disease. In: The genetic basis of common diseases. Oxford: Oxford University Press; 1992. p. 222-39.

7. Zorzetto M, Russi E, Senn O, Imboden M, Ferrarotti I, Tinelli C, et al. SERPINA1 gene variants in individuals from the general population with reduced alpha1-antitrypsin concentrations. Clin Chem. 2008;54:1331-8.

8. Ferrarotti I, Thun GA, Zorzetto M, Ottaviani S, Imboden M, Schindler C, et al. Serum levels and genotype distribution of alpha1-antitrypsin in the general population. Thorax. 2012;67:669-74.

9. Bashir A, Shah NN, Hazari YM, Habib M, Bashir S, Hilal N, et al. Novel variants of SERPIN1A gene: interplay between alpha1-antitrypsin deficiency and chronic obstructive pulmonary disease. Respir Med. 2016;117:139-49.

10. Kueppers F, Sanders C. State-of-the-art testing for alpha-1 antitrypsin deficiency. Allergy Asthma Proc. 2017;38:108-14.

11. Rizzo JM, Buck MJ. Key principles and clinical applications of "nextgeneration" DNA sequencing. Cancer Prev Res (Phila). 2012;5:887-900.

12. Graham RP, Dina MA, Howe SC, Butz ML, Willkomm KS, Murray DL, et al SERPINA1 full-gene sequencing identifies rare mutations not detected in targeted mutation analysis. J Mol Diagn. 2015;17:689-94.

13. Robert $X$, Gouet P. Deciphering key features in protein structures with the new ENDscript server. Nucleic Acids Res. 2014;42:W320-4

14. Wei $Q, X u Q$, Dunbrack RL Jr. Prediction of phenotypes of missense mutations in human proteins from biological assemblies. Proteins. 2013;81:199-213.

15. Schymkowitz J, Borg J, Stricher F, Nys R, Rousseau F, Serrano L. The FoldX web server: an online force field. Nucleic Acids Res. 2005;33:W382-8.

16. Tokuriki N, Stricher F, Serrano L, Tawfik DS. How protein stability and new functions trade off. PLoS Comput Biol. 2008;4:e1000002.

17. Adzhubei IA, Schmidt S, Peshkin L, Ramensky VE, Gerasimova A, Bork P, et al. A method and server for predicting damaging missense mutations. Nat Methods. 2010;7:248-9.

18. National Center for Biotechnology Information. ClinVar database. SERPINA1 [gene]. Available at: https://www.ncbi.nlm.nih.gov/clinvar/?term= SERPINA1\%5Bgene\%5D. Accessed on: 18 Dec 2017.

19. Giacopuzzi E, Laffranchi M, Berardelli R, Ravasio V, Ferrarotti I, Gooptu B, et al. Realworld clinical applicability of pathogenicity predictors assessed on SERPINA1 mutations in alpha-1-antitrypsin deficiency. Hum Mutat. 2018:39:1203-13.

20. Silva D, Oliveira MJ, Guimaraes M, Lima R, Gomes S, Seixas S. Alpha-1antitrypsin (SERPINA1) mutation spectrum: three novel variants and haplotype characterization of rare deficiency alleles identified in Portugal. Respir Med. 2016;116:8-18

21. Fra AM, Gooptu B, Ferrarotti I, Miranda E, Scabini R, Ronzoni R, et al. Three new alpha1-antitrypsin deficiency variants help to define a C-terminal region regulating conformational change and polymerization. PLoS One. 2012;7:e38405

22. Joly P, Lacan P, Chapuis-Cellier C, Garcia C, Bererd M, Francina A. Molecular characterization of 7 new alpha-1 anti-trypsin (A1AT) variants including two with an associated deficient phenotype. Clin Chim Acta. 2014;427:21-2.

23. Arora NK, Arora S, Ahuja A, Mathur P, Maheshwari M, Das MK, et al. Alpha 1 Antitrypsin Deficiency in children with chronic liver disease in North India. Indian Pediatr. 2010:47:1015-23.

24. Zhan SH, Abboud RT, Jung B, Kuchinka B, Ralston D, Casey B, et al. Sanger sequencing solved a cryptic case of severe alpha (1)-antitrypsin deficiency. Clin Biochem. 2012;45:499-501

25. Ferrarotti I, Poplawska-Wisniewska B, Trevisan MT, Koepke J, Dresel M, Koczulla R, et al. How can we improve the detection of Alpha1-antitrypsin deficiency? PLoS One. 2015;10:e0135316.

26. Matamala N, Lara B, Gomez-Mariano G, Martinez S, Retana D, Fernandez T, et al. Characterization of novel missense variants of SERPINA1 gene causing Alpha-1 antitrypsin deficiency. Am J Respir Cell Mol Biol. 2018;58:706-16. 
27. Schievink WI, Katzmann JA, Piepgras DG, Schaid DJ. Alpha-1-antitrypsin phenotypes among patients with intracranial aneurysms. J Neurosurg. 1996; 84:781-4.

28. Sandhaus RA, Turino G, Brantly ML, Campos M, Cross CE, Goodman K, et al. The diagnosis and Management of Alpha-1 antitrypsin deficiency in the adult. Chronic Obstr Pulm Dis (Miami). 2016;3:668-82.

29. Sinden NJ, Koura F, Stockley RA. The significance of the F variant of alpha-1antitrypsin and unique case report of a PiFF homozygote. BMC Pulm Med. 2014;14:132.

30. Molloy K, Hersh CP, Morris VB, Carroll TP, O'Connor CA, Lasky-Su JA, et al. Clarification of the risk of chronic obstructive pulmonary disease in alantitrypsin deficiency PiMZ heterozygotes. Am J Respir Crit Care Med. 2014; 189:419-27.

31. Graham A, Kalsheker NA, Newton CR, Bamforth FJ, Powell SJ, Markham AF. Molecular characterisation of three alpha-1-antitrypsin deficiency variants: proteinase inhibitor (pi) nullcardiff (Asp256----Val); PiMmalton (Phe51---deletion) and Pil (Arg39----Cys). Hum Genet. 1989;84:55-8.

32. Chapman KR, Burdon JG, Piitulainen E, Sandhaus RA, Seersholm N, Stocks $J M$, et al. Intravenous augmentation treatment and lung density in severe alpha1 antitrypsin deficiency (RAPID): a randomised, double-blind, placebocontrolled trial. Lancet. 2015;386:360-8.

33. McElvaney NG, Burdon JG, Holmes M, Glanville A, Wark PAB, Thompson PJ, et al. Long-term efficacy and safety of a1 proteinase inhibitor treatment for emphysema caused by severe a1 antitrypsin deficiency: an open-label extension trial (RAPID-OLE). Lancet Respir Med. 2017;5:51-60.

34. Stoller JK, Sandhaus RA, Turino G, Dickson R, Rodgers K, Strange C. Delay in diagnosis of alpha1-antitrypsin deficiency: a continuing problem. Chest. 2005b;128:1989-94.

35. Ringenbach MR, Banta E, Snyder MR, Craig TJ, Ishmael FT. A challenging diagnosis of alpha-1-antitrypsin deficiency: identification of a patient with a novel F/null phenotype. Allergy Asthma Clin Immunol. 2011;7:18.

\section{Publisher's Note}

Springer Nature remains neutral with regard to jurisdictional claims in published maps and institutional affiliations.

Ready to submit your research? Choose BMC and benefit from:

- fast, convenient online submission

- thorough peer review by experienced researchers in your field

- rapid publication on acceptance

- support for research data, including large and complex data types

- gold Open Access which fosters wider collaboration and increased citations

- maximum visibility for your research: over $100 \mathrm{M}$ website views per year

At $\mathrm{BMC}$, research is always in progress.

Learn more biomedcentral.com/submissions 